\title{
SURFACE WAVE CHARACTERISTICS IN A MICROPOLAR TRANSVERSELY ISOTROPIC HALFSPACE UNDERLYING AN INVISCID LIQUID LAYER
}

\author{
R.R. GUPTA* \\ Department of Mathematics and Applied Sciences \\ MEC, OMAN \\ E-mail: dr.rajanigupta@gmail.com \\ R.R. GUPTA \\ Department of Mathematics, MMU \\ Mullana, INDIA
}

\begin{abstract}
The present investigation deals with the propagation of waves in a micropolar transversely isotropic half space with an overlying inviscid fluid layer. Effects of fluid loading and anisotropy on the phase velocity, attenuation coefficient, specific loss and relative frequency shift. Finally, a numerical solution was carried out for aluminium epoxy material and the computer simulated results for the phase velocity, attenuation coefficient, specific loss and relative frequency shift are presented graphically. A particular case for the propagation of Rayleigh waves in a micropolar transversely isotropic half-space is deduced and dispersion curves are plotted for the same as functions of the wave number. An amplitude of displacements and microrotation together with the path of surface particles are also calculated for the propagation of Rayleigh waves in the latter case.
\end{abstract}

Key words: micropolar, transversely isotropic, amplitude ratios, Rayleigh waves.

\section{Introduction}

In the classical theory of elasticity the microstructure of a material is not taken into consideration for studying the mechanical behavior of the material due to external stimuli. But discrepancies are observed in the classical theory and experimental results while studying the stress concentration in the neighborhood of holes and cracks, especially in the material containing laminates, granules, fibers. The discrepancy indicates that the material response to external stimuli depends on the motions of inner-structures and so the study of a micropolar elastic medium is necessary.

Suhubi and Eringen (1964) developed a non-linear theory of microelastic solids in which the micromotions of the material particles contained in a macrovolume element with respect to its centroid are taken into account. Materials affected by such micromotions and microdeformations are called micromorphic materials. Eringen (1966) developed theories for a subclass of micromorphic materials which are called micropolar media and these materials show microrotational effects and microrotational inertia. Here, the material particles in a volume element can undergo only rigid rotational motions about their center of mass. The motion described here does not only consists of deformation but also of microrotation giving six degrees of freedom. The interaction between two parts of a body is transmitted not only by a force but also by a torque, resulting in asymmetric force stresses and couple stresses. Physically, solid propellant grains, polymeric materials and fiber glass are examples for such materials. The theory is expected to find

\footnotetext{
* To whom correspondence should be addressed
} 
applications in mechanics of granular materials, composites fibrous materials and particularly microcracks and microfractures.

Eringen's micropolar theory is more appropriate for geological materials like rocks, soils, etc., since this theory takes into account the intrinsic rotation and predicts the behavior of a material with an inner structure. Many investigators discussed different types of problems in transversely isotropic elastic materials. Abubakar (1962) discussed free vibrations of a transversely isotropic plate. Keck et al. (1971) derived the frequency equation for the propagation of train of nontorsional axisymmetric harmonic wave in infinitely long shells, made of three concentric cylinders of different transversely isotropic materials. In 1974, Shuvalov et al. described the long wavelength onset of the fundamental branches for a free anisotropic plate with an arbitrary variation of material properties. Payton in 1991 studied wave propagation in a restricted transversely isotropic elastic solid whose slowness surface contains conical points. In spite of these studies, no attempt has been made to study wave propagation in a micropolar transversely isotropic medium.

The aim of the present study is to improve our knowledge about the propagation of Rayleigh waves in a micropolar transversely isotropic medium underlying a layer of an inviscid liquid of finite thickness. This study has many applications in various fields of science and technology, namely, atomic physics, industrial engineering, thermal power plants, submarine structures, pressure vessel, aerospace, chemical pipes and metallurgy. After developing the solution, frequency equations connecting the phase velocity with the wave number are derived. The curves of phase velocity, attenuation coefficient, specific loss and relative frequency shift are presented and illustrated graphically, to indicate the effect of anisotropy and thickness of fluid loading. The propagation of Rayleigh waves together with the path of surface particles in a micropolar transversely isotropic half-space are also deduced from the present investigation.

\section{Basic equations}

Following Eringen (1999), the constitutive relations and balance laws in a general micropolar anisotropic medium possessing a center of symmetry, in the absence of body forces, body couples, are given by

\section{Constitutive relations}

$$
\begin{aligned}
& t_{i j}=A_{i j k l} E_{k l}+G_{i j k l} \Psi_{k l}, \\
& m_{i j}=G_{i j k l} E_{k l}+B_{i j k l} \Psi_{k l}
\end{aligned}
$$

The deformation and wryness tensor are defined by

$$
E_{j i}=u_{i, j}+\varepsilon_{i j k l} \varphi_{k}, \quad \Psi_{i j}=\varphi_{i, j}
$$

\section{Balance laws}

$$
\begin{aligned}
& t_{i j, j}=\rho \ddot{u}_{i} \\
& m_{i k, i}+\varepsilon_{i j k} t_{i j}=\rho j \ddot{\varphi}_{k},
\end{aligned}
$$

where the list of symbols is given in the nomenclature.

The basic equation which governs the motion of an inviscid liquid, given by Ewing et al. (1957) is 


$$
\lambda_{o} \nabla\left(\nabla \cdot \bar{u}^{o}\right)=\rho_{o} \frac{\partial^{2} \bar{u}^{o}}{\partial t^{2}}
$$

The stress displacement relation is given by

$$
t_{i j}^{o}=\lambda_{o} u_{r, r}^{o} \delta_{i j}
$$

where the list of symbols is given in the nomenclature.

\section{Problem formulation and solution}

Following Slaughter (2002), appropriate transformations have been used on the set of Eqs (2.1) to derive equations for a micropolar transversely isotopic medium and restrict our analysis to a two dimensional problem.

In the present paper, we consider a layer of an inviscid liquid, of finite thickness $H$ with traction free surface at $x_{3}=-H$, overlying a homogeneous, micropolar transversely isotropic half-space. The origin of the coordinate system $\left(x_{1}, x_{2}, x_{3}\right)$ is taken at any point of the plane surface (interface) and the $x_{3}$-axis points vertically downwards into the solid half-space which is thus represented by $x_{3} \geq 0$. We consider the propagation of waves in the direction of the $x_{1}$-axis such that all the particles on a line parallel to the $x_{2}$-axis are equally displaced. Therefore all the parameters become independent of the $x_{2}$-coordinate. Further, it is assumed that the disturbances are small and are confined to the neighborhood of the interface $x_{3}=0$ and hence vanish as $x_{3} \rightarrow \infty$. For the two-dimensional problem, we assume the components of the displacement and microrotation vector in the inviscid layer and micropolar transversely isotropic half-space of the form

$$
\boldsymbol{u}^{o}=\left(u_{1}^{o}, 0, u_{3}^{o}\right), \quad \boldsymbol{u}=\left(u_{1}, 0, u_{3}\right), \quad \boldsymbol{\varphi}=\left(0, \varphi_{2}, 0\right)
$$

and assume that the solutions are explicitly independent of $x_{2}$, i.e., $\frac{\partial}{\partial x_{2}}=0$. Thus the field equations reduce to

$$
\begin{aligned}
& A_{11} \frac{\partial^{2} u_{1}}{\partial x_{1}^{2}}+A_{55} \frac{\partial^{2} u_{1}}{\partial x_{3}^{2}}+\left(A_{13}+A_{56}\right) \frac{\partial^{2} u_{3}}{\partial x_{1} \partial x_{3}}+K_{1} \frac{\partial \varphi_{2}}{\partial x_{3}}=\rho \frac{\partial^{2} u_{1}}{\partial t^{2}} \\
& A_{66} \frac{\partial^{2} u_{3}}{\partial x_{1}^{2}}+A_{33} \frac{\partial^{2} u_{3}}{\partial x_{3}^{2}}+\left(A_{13}+A_{56}\right) \frac{\partial^{2} u_{1}}{\partial x_{1} \partial x_{3}}+K_{2} \frac{\partial \varphi_{2}}{\partial x_{1}}=\rho \frac{\partial^{2} u_{3}}{\partial t^{2}} \\
& B_{77} \frac{\partial^{2} \varphi_{2}}{\partial x_{1}^{2}}+B_{66} \frac{\partial^{2} \varphi_{2}}{\partial x_{3}^{2}}-K_{1} \frac{\partial u_{1}}{\partial x_{3}}+K_{1} \frac{\partial \varphi_{2}}{\partial x_{3}}=\rho \frac{\partial^{2} u_{1}}{\partial t^{2}}, \\
& \lambda_{o} \frac{\partial}{\partial x_{1}}\left(\frac{\partial u_{1}^{o}}{\partial x_{1}}+\frac{\partial u_{3}^{o}}{\partial x_{3}}\right)=\rho_{o} \frac{\partial^{2} u_{1}^{o}}{\partial t^{2}},
\end{aligned}
$$




$$
\begin{aligned}
& \lambda_{o} \frac{\partial}{\partial x_{3}}\left(\frac{\partial u_{1}^{o}}{\partial x_{1}}+\frac{\partial u_{3}^{o}}{\partial x_{3}}\right)=\rho_{o} \frac{\partial^{2} u_{3}^{o}}{\partial t^{2}}, \\
& t_{33}=A_{11} \frac{\partial u_{1}}{\partial x_{1}}+A_{33} \frac{\partial u_{3}}{\partial x_{3}}, \\
& t_{31}=A_{65} \frac{\partial u_{3}}{\partial x_{1}}-K_{1} \varphi_{2}+A_{55} \frac{\partial u_{1}}{\partial x_{3}}, \quad m_{32}=B_{66} \frac{\partial \varphi_{2}}{\partial x_{3}}, \\
& t_{33}^{o}=\lambda_{o}\left(\frac{\partial u_{1}^{o}}{\partial x_{1}}+\frac{\partial u_{3}^{o}}{\partial x_{3}}\right)
\end{aligned}
$$

where

$$
K_{1}=A_{56}-A_{55}, \quad K_{2}=A_{66}-A_{56}, \quad X=K_{2}-K_{1},
$$

and we have used the notations $11 \rightarrow 1,33 \rightarrow 3,12 \rightarrow 7,13 \rightarrow 6,23 \rightarrow 5$ for the material constants.

For further considerations, it is convenient to introduce the dimensionless variables defined by

$$
\begin{aligned}
& \left(x_{1}^{\prime}, x_{3}{ }^{\prime}\right)=\frac{\omega^{*}}{c_{1}}\left(x_{1}, x_{3}\right), \quad\left(u_{1}{ }^{\prime}, u_{3}{ }^{\prime}\right)=\frac{\omega^{*}}{c_{1}}\left(u_{1}, u_{3}\right), \quad t_{i j}^{\prime}=\frac{t_{i j}}{A_{55}}, \\
& m_{i j}^{\prime}=\frac{m_{i j} c_{1}}{B_{56} \omega^{*}}, \quad \varphi_{2}^{\prime}=\frac{\varphi_{2} A_{55}}{K_{1}}, \quad t^{\prime}=\omega^{*} t, \quad \omega^{* 2}=\frac{X}{\rho j}, \quad c_{1}^{2}=\frac{A_{55}}{\rho} .
\end{aligned}
$$

\section{Boundary condition}

The boundary conditions are:

(i) The upper boundary of the layer is assumed to be stress free. Therefore, the normal stress vanishes at the free surface $x_{3}=-H$, i.e.,

$$
t_{33}^{o}=0, \quad \text { at } \quad x_{3}=-H
$$

(ii) Continuity of normal stress at the interface $x_{3}=0$ between the liquid layer and the micropolar transversely isotropic half-space, i.e.,

$$
t_{33}=t_{33}^{0}
$$

(iii) Continuity of normal displacement at the interface $x_{3}=0$ between the liquid layer and the micropolar transversely isotropic half-space, i.e.,

$$
u_{3}=u_{3}^{o}
$$


(iii) Vanishing of the tangential stress component at the interface $x_{3}=0$ between the liquid layer and the micropolar transversely isotropic half-space, i.e.,

$$
t_{31}=0
$$

(iv) Vanishing of the tangential couple stress component at the interface $x_{3}=0$ between the liquid layer and the micropolar transversely isotropic half-space, i.e.,

$$
m_{32}=0 .
$$

\section{Normal mode analysis and solution of the problem}

We assume the solution for $\bar{u}_{1}, \bar{u}_{3}, \bar{\varphi}_{2}, \bar{u}_{1}^{o}, \bar{u}_{3}^{o}$ representing propagating waves in the $x_{1}-x_{3}$ plane of the form

$$
\left(u_{1}, u_{3}, \varphi_{2}\right)=\left(\bar{u}_{1}, \bar{u}_{3}, \bar{\varphi}_{2}\right) e^{i \xi\left(x_{1}+m x_{3}-c t\right)}, \quad\left(u_{1}^{o}, u_{3}^{o}\right)=\left(\bar{u}_{3}^{o}, \bar{u}_{3}^{o}\right) e^{i \xi\left(x_{1}+m x_{3}-c t\right)}
$$

where $\xi$ is the wave number, $\omega=\xi_{c}$ is the angular frequency and $c$ is the phase velocity of the wave, $m$ is the unknown parameter which signifies the penetration depth of the wave, $\bar{u}_{3}, \bar{\varphi}_{2}$ are respectively, the amplitude ratios of the displacement $u_{3}$ and microrotation $\varphi_{2}$ with respect to the displacement $u_{1}$.

With the help of Eqs (3.10) and (5.1), field Eqs (3.2)-(3.6) reduced to (after suppressing primes)

$$
\begin{aligned}
& \left\{\left[-m^{2}+\left(c^{2}-d_{1}\right)\right] \bar{u}_{1}-m\left(d_{1}+d_{2}\right) \bar{u}_{3}+i \xi m\left(d_{2}-1\right) \bar{\varphi}_{2}\right\} e^{i \xi\left(x_{1}+m x_{3}-c t\right)}=0 \\
& {\left[-m \xi^{2}\left(d_{1}+d_{2}\right) \bar{u}_{1}+\left(m^{2}-d_{3}+d_{4} c^{2}\right) \xi^{2} \bar{u}_{3}+i \xi\left(d_{2}-1\right)\left(d_{3}-d_{2} d_{4}\right) \bar{\varphi}_{2}\right] u_{1} e^{i \xi\left(x_{1}+m x_{3}-c t\right)}=0} \\
& {\left[i \xi m d_{7} \bar{u}_{1}+\frac{i \xi d_{7}\left(d_{3}-d_{2} d_{4}\right)}{d_{4}\left(d_{2}-1\right)} \bar{u}_{3}+\left[\left(m^{2}-d_{5}-c^{2} d_{6}\right) \xi^{2}+d_{8}\right] \bar{\varphi}_{2}\right] u_{1} e^{i \xi\left(x_{1}+m x_{3}-c t\right)}=0} \\
& \left(1-\frac{\rho_{o} c_{1}^{2}}{\lambda_{o}} c^{2}\right) \bar{u}_{1}^{o}+m u_{3}^{o}=0
\end{aligned}
$$

where

$$
\begin{aligned}
& d_{1}=A_{11} / A_{55}, \quad d_{2}=A_{56} / A_{55}, \quad d_{3}=A_{66} / A_{55}, \quad d_{4}=A_{55} / A_{33}, \quad d_{5}=B_{77} / B_{66}, \\
& d_{6}=A_{55} j / B_{66}, \quad d_{7}=A_{55} c_{1}^{2} / B_{66} \omega^{* 2}, \quad d_{8}=K_{1} / A_{55}, \quad d_{9}=A_{13} / A_{55} .
\end{aligned}
$$

The condition for the non trivial solution of the system of Eqs (5.2) yields a cubic equation in $m^{2}$ as

$$
A m^{6}+B m^{4}+C m^{2}+D=0
$$


where

$$
\begin{aligned}
& A=-\xi^{2}, \quad B=-\xi^{2}\left[d_{3}+d_{5}+d_{1}-c^{2}-\left(d_{1}+d_{2}\right)^{2}\right]+\omega^{2}\left(d_{6}+d_{4}\right)-d_{8}+d_{7}\left(d_{2}-1\right)^{2}, \\
& C=a_{2} d_{7}-a_{1}\left(c^{2} d_{4}-d_{3}\right)-\left(c^{2}-d_{1}\right)\left(a_{1}-a_{3} \xi^{2}\right)-a_{1}\left(d_{1}+d_{2}\right)^{2}+ \\
& -a_{4}\left[\left(d_{1}+d_{2}\right)\left(d_{3}-d_{2} d_{4}\right) a_{5}+\left(d_{2}-1\right) a_{3}\right], \\
& D=\left(c^{2}-d_{1}\right)\left(c^{2} d_{4}-d_{3}\right) a_{1}-d_{7} a_{2}\left(c^{2}-d_{1}\right), \quad a_{1}=\omega^{2} d_{6}-d_{5} \xi^{2}-d_{8}, \quad a_{2}=\frac{\left(d_{3}-d_{2} d_{4}\right)^{2}}{d_{4}}, \\
& a_{3}=d_{3}-c^{2} d_{4}, \quad a_{4}=d_{7}\left(d_{2}-1\right), \quad a_{5}=\frac{\left(d_{4}+1\right)}{d_{4}}, \quad a_{6}=a_{2}, \quad a_{7}=\frac{\left(d_{1}-d_{2}\right)}{\left(d_{2}-1\right)} .
\end{aligned}
$$

The roots of this equation give three values of $m^{2}$, and hence of $c^{2}$. Three positive values of $c$ will be the velocities of propagation of three possible waves, viz. the quasi-longitudinal displacement (QLD) wave, transverse displacement (QCTD) wave and quasi-coupled quasi-coupled transverse microrotational (QCTM) wave. This fact is verified, when we solve Eq.(3.10), using Matlab programming. For the isotropic linear micropolar elastic solid, if we put, i.e.

$$
\begin{array}{ll}
A_{11}=A_{33}=\lambda+2 \mu+K, & A_{55}=A_{66}=\mu+K, \quad A_{13}=\lambda, A_{56}=\mu, \\
K=-K_{1}=K_{2}=X / 2, \quad B_{66}=B_{77}=\gamma
\end{array}
$$

in Eq.(5.4), the velocity $c_{1}$ corresponds to the longitudinal displacement wave and the velocities $c_{2}$ and $c_{3}$ correspond to two coupled waves, viz. the transverse microrotational and transverse displacement wave as obtained by Parfitt and Eringen (1969).

So Eq.(3.10) leads to the following solution for displacements and microrotation as

$$
\left(\bar{u}_{1}, \bar{u}_{3}, \bar{\varphi}_{2}\right)=\sum_{i=1}^{3} A_{i} \cos \left(m_{i} x_{3} \xi\right)+B_{i} \sin \left(m_{i} x_{3} \xi\right) e^{i \xi\left(x_{1}-c t\right)}
$$

where

$$
r_{i}=\frac{m_{i}\left[\left(m_{i}^{2} \xi^{2}-a_{1}\right)\left(d_{1}+d_{2}\right)-a_{4}\left(d_{3}-d_{2} d_{4}\right)\right]}{m_{i}^{4} \xi^{2}-m_{i}^{2}\left(a_{1}-\xi^{2} a_{3}\right)-a_{3} a_{1}-a_{6}}, \quad t_{i}=\frac{i m_{i} d_{7}\left(a_{7} a_{6}-m_{i}^{2}-a_{3}\right)}{m_{i}^{4} \xi^{2}-m_{i}^{2}\left(a_{1}-\xi^{2} a_{3}\right)-a_{3} a_{1}-a_{6}} .
$$

Similarly, Eq.(5.3) leads to the following solution for displacement components of the inviscid liquid layer of the form

$$
\left(u_{1}^{o}, u_{3}^{o}\right)=A_{4} \cos \left(m_{4} x_{3} \xi\right)+B_{4} \sin \left(m_{4} x_{3} \xi\right)\left(1, r_{4}\right) e^{i \xi\left(x_{1}-c t\right)}
$$

where

$$
m_{4}^{2}=b_{1} c^{2}-1, \quad r_{4}=-\frac{m_{4}}{b_{1} c^{2}-1}, \quad b_{1}=\frac{\rho_{o} c_{1}^{2}}{\lambda_{o}}
$$




\section{Derivation of secular equation}

Substituting the values of $\bar{u}_{1}, \bar{u}_{3}$ and $\bar{\varphi}_{2}$ from Eq.(5.1) in the boundary conditions (4.1)-(4.5), and equating the determinant of coefficients of $A i$ and $B i i=1,2,3,4$ of the resulting equations to zero, we obtain an equation for the frequency of the present system. Thus, the frequency equation, after applying lengthy algebraic reductions and manipulations of the determinant leads to the following secular equation

$$
c^{2}=\frac{A_{55}^{2} b_{5}^{*}\left(a+a_{4}^{*}\right)^{2}+\lambda_{o}^{2} \xi^{2} b_{4}^{* 2}}{\lambda_{o}^{2} \xi^{2} b_{4}^{* 2} b_{1}^{* 2}},
$$

where

$$
\begin{aligned}
& a_{i}^{*}=d_{1} i \xi-\frac{m_{i} r_{i} \xi}{d_{4}}, \quad c_{i}^{*}=d_{2} i \xi r_{i}-m_{i} \xi+\left(d_{2}-1\right)^{2} s_{i}, \quad i=1,2,3, \quad a_{4}^{*}=\frac{\lambda_{o}}{A_{55}} i \xi r_{4}, \\
& a_{5}^{*}=\frac{\lambda_{0}}{A_{55}} i \xi m_{4}, \quad \frac{\lambda_{0}}{A_{55}} i \xi, \quad b_{4}^{*}=i \xi r_{4} \cos \left(m_{4} \xi H\right)+m_{4} \xi \sin \left(m_{4} \xi H\right), \\
& b_{5}^{*}=-i \xi r_{4} \sin \left(m_{4} \xi H\right)+m_{4} \xi \cos \left(m_{4} \xi H\right), \quad c_{i}=\cos \left(m_{i} \xi x_{3}\right), \\
& a=\frac{m_{1} s_{1}\left(a_{2}^{*} c_{3}^{*}-a_{3}^{*} c_{2}^{*}\right)+m_{2} s_{2}\left(a_{3}^{*} c_{1}^{*}-a_{1}^{*} c_{3}^{*}\right)+m_{3} s_{3}\left(\left(a_{1}^{*} c_{2}^{*}-a_{2}^{*} c_{1}^{*}\right)\right.}{m_{1} s_{1}\left(r_{2} c_{3}^{*}-r_{3} c_{2}^{*}\right)+m_{2} s_{2}\left(r_{3} c_{1}^{*}-r_{1} c_{3}^{*}\right)+m_{3} s_{3}\left(r_{1} c_{2}^{*}-r_{2} c_{1}^{*}\right)}
\end{aligned}
$$

\subsection{Specific loss}

The specific loss is the ratio of energy $(\Delta W)$ dissipated in taking a specimen through a stress cycle to the elastic energy $(W)$ stored in the specimen when the strain is maximum. Kolsky (1963) shows that the specific loss $(\Delta W / W)$ is $4 \pi$ times the absolute value of the ratio of the imaginary part of the wave number to the real part of the wave number, i.e.

$$
\frac{\Delta W}{W}=4 \pi\left|\frac{\operatorname{Im}(k)}{\operatorname{Re}(k)}\right| .
$$

He noted that the specific loss is the most direct method of defining internal friction for a material.

\subsection{Relative frequency shift}

The relative frequency shift is defined by the relation as given below

$$
R F S=\left|\frac{\omega\left(\rho_{L}\right)-\omega(0)}{\omega(0)}\right|
$$

where $\omega\left(\rho_{L}\right), \omega(0)$ are the frequencies in the presence and absence of fluid loading, respectively. 


\subsection{Particular case:}

\section{Rayleigh wave equation}

In this case, the boundary conditions reduced to

$$
t_{33}=0, \quad t_{31}=0, \quad m_{32}=0 \quad \text { at } \quad x_{3}=0 .
$$

Substituting the values of stress components from Eqs (3.7) and (3.8) in boundary condition (6.3), we get

$$
\sum_{i=1}^{3} A_{i} a_{i}^{*}=0, \quad \sum_{i=1}^{3} A_{i} c_{i}^{*}=0
$$

where $a_{i}^{*}$ and $c_{i}^{*}$ are the same as defined above in Eqs (6.2).

The three boundary conditions given by Eqs (6.3) are satisfied simultaneously if the determinant of the coefficients of $A_{i} i=1,2,3$, vanishes in Eqs (6.4). So we obtain the frequency equation for the Rayleigh waves in the micropolar transversely isotropic medium of the form

$$
c^{2}=\frac{b^{2}+1}{m_{3}^{2}+1} \quad \text { where } \quad b=-\left[m_{1} s_{1}\left(a_{2}^{*} c_{3}^{*}-a_{3}^{*} c_{2}^{*}\right)+m_{2} s_{2}\left(a_{3}^{*} c_{1}^{*}-a_{1}^{*} c_{3}^{*}\right)\right] \text {. }
$$

\section{Numerical results and discussion}

In order to illustrate theoretical results obtained in the preceding sections, we now present some numerical results taking

$$
\begin{aligned}
& A_{11}=13.8 \times 10^{10} \mathrm{Nm}^{-2}, \quad A_{33}=14.43 \times 10^{10} \mathrm{Nm}^{-2}, \quad A_{55}=3.7 \times 10^{10} \mathrm{Nm}^{-2}, \\
& A_{66}=4.2 \times 10^{10} \mathrm{Nm}^{-2}, \quad A_{13}=8.85 \times 10^{10} \mathrm{Nm}^{-2}, \quad A_{56}=2.977 \times 10^{10} \mathrm{Nm}^{-2}, \\
& B_{77}=3.71 \times 10^{9} \mathrm{~N}, \quad B_{66}=3.9 \times 10^{9} \mathrm{~N} .
\end{aligned}
$$

For a comparison with a micropolar isotropic solid, following Gauthier (1982), we take the following values of the relevant parameters for the case of an aluminium epoxy composite as,

$$
\begin{array}{ll}
\rho=2.19 \times 10^{3} \mathrm{Kgm}^{-3}, & \lambda=7.59 \times 10^{9} \mathrm{Nm}^{-2}, \quad \mu=1.89 \times 10^{10} \mathrm{Nm}^{-2}, \\
K=1.49 \times 10^{9} \mathrm{Nm}^{-2}, & \gamma=2.63 \times 10^{9} \mathrm{~N}, \quad j=0.196 \times 10^{-2} \mathrm{~m}^{2} .
\end{array}
$$

The non-dimensional phase velocity, attenuation coefficient, specific loss and relative frequency shift (RFS) were computed and presented graphically (Figs 1-4) for various values of the non-dimensional wave number to compare the results for the cases of the micropolar transversely isotropic solid (MTIS), micropolar isotropic solid (MIS) and for two different values of thickness $H=.01$ and $H=1$ of the inviscid liquid layer. In all the figures, the solid curves represent the variations corresponding to the micropolar transversely isotropic solid (MTIS), while the broken curves represent the variations corresponding to the 
micropolar isotropic solid (MIS). Also, the curves without a center symbol correspond to the variation when $H=.01$, whereas the curves with the center symbol (-O-O-) correspond to the variation when $H=1$. Figures 57 represent, respectively, the variation of phase velocity, attenuation coefficient and specific loss with respect to the wave number for the solid half-space(in the absence of the liquid layer) to compare the results for different cases MTIS and MIS. The plots of amplitudes of normal displacement, tangential displacement and microrotation are shown in Figs 8-10. In these figures, solid line represents the variations for MTIS, while broken lines represent the variations for MIS.

It is shown in Fig. 1 that the value of phase velocity at $H=.01$, initially decreases within the interval $(0,1.5)$ and then increases slowly to attain a constant value. However, for $H=1$, it oscillates with an oscillating amplitude to attain a constant value at the end, in both the cases of MTIS and MIS.
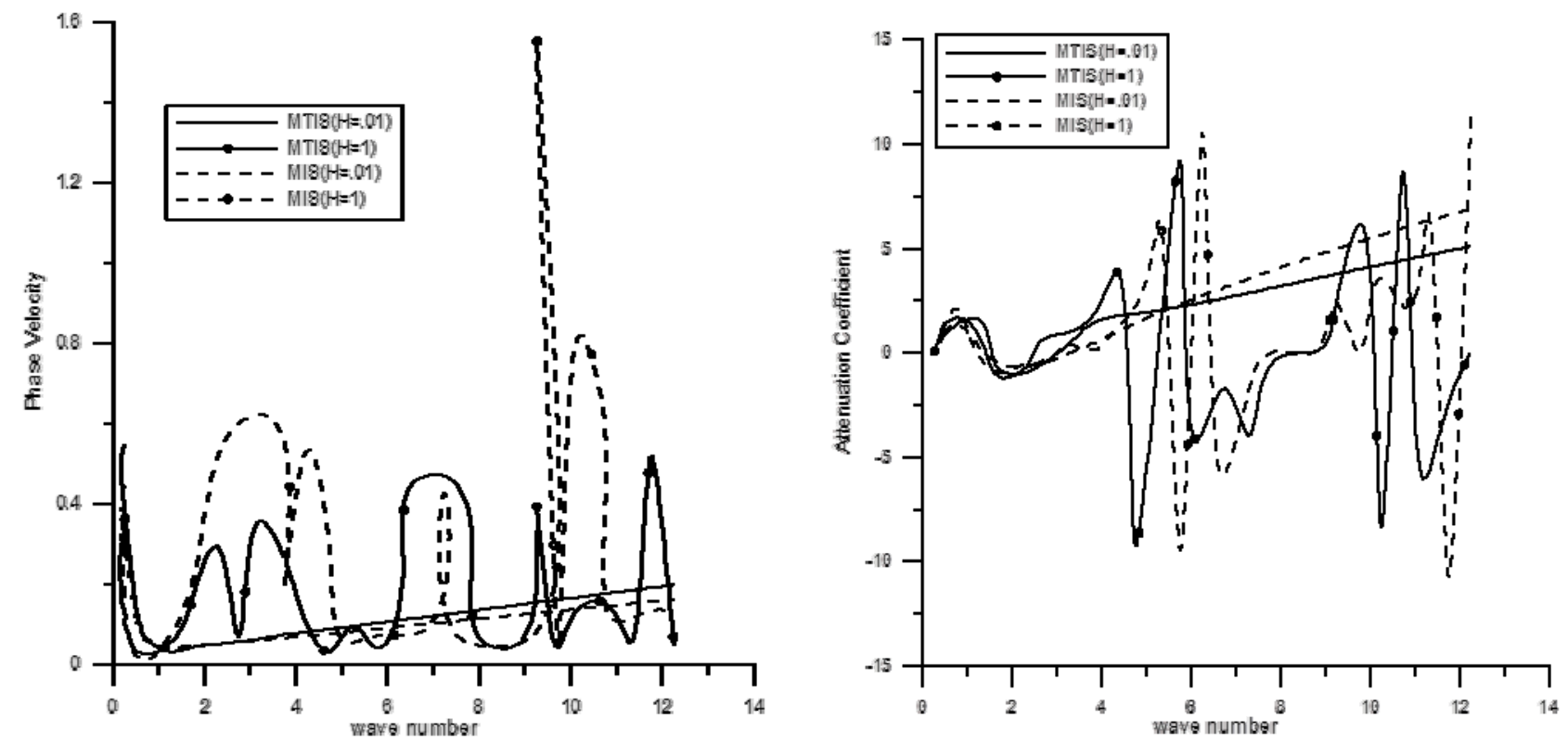

Fig.1. Variation of phase velocity with wave number. Fig.2. Variation of attenuation coefficient with wave number.
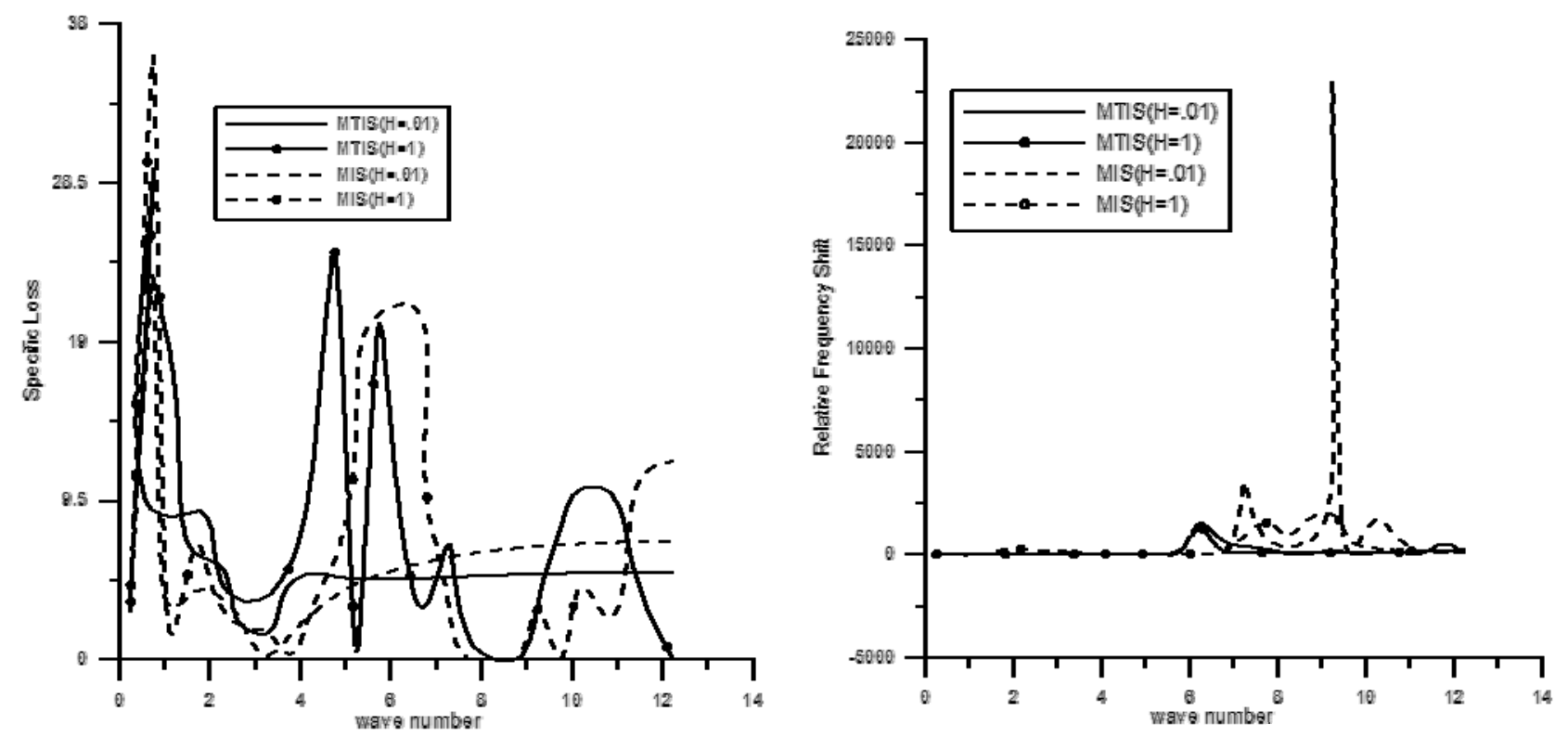

Fig.3. Variation of specific loss with wave number. Fig.4. Variation of relative frequency shift with wave number. 
The variation of the attenuation coefficient and specific loss with respect to the wave number is indicated in Figs 2 and 3, respectively. It is seen from these figures that for $H=.01$, and for both the cases of MTIS and MIS, the value of the attenuation coefficient and specific loss initially oscillates, then increases to become constant, while for $H=1$, their values oscillate with varying amplitude. It is also observed that the values of phase velocity, attenuation coefficient and specific loss are not much affected by anisotropy, but an appreciable effect of thickness of the liquid layer is observed. It follows from Fig. 4 that the value of relative frequency shift initially appears to be constant and then oscillates to become a constant for MTIS, while for MIS its value is initially constant and shows a peak value when $H=.01$, to become constant at the end.
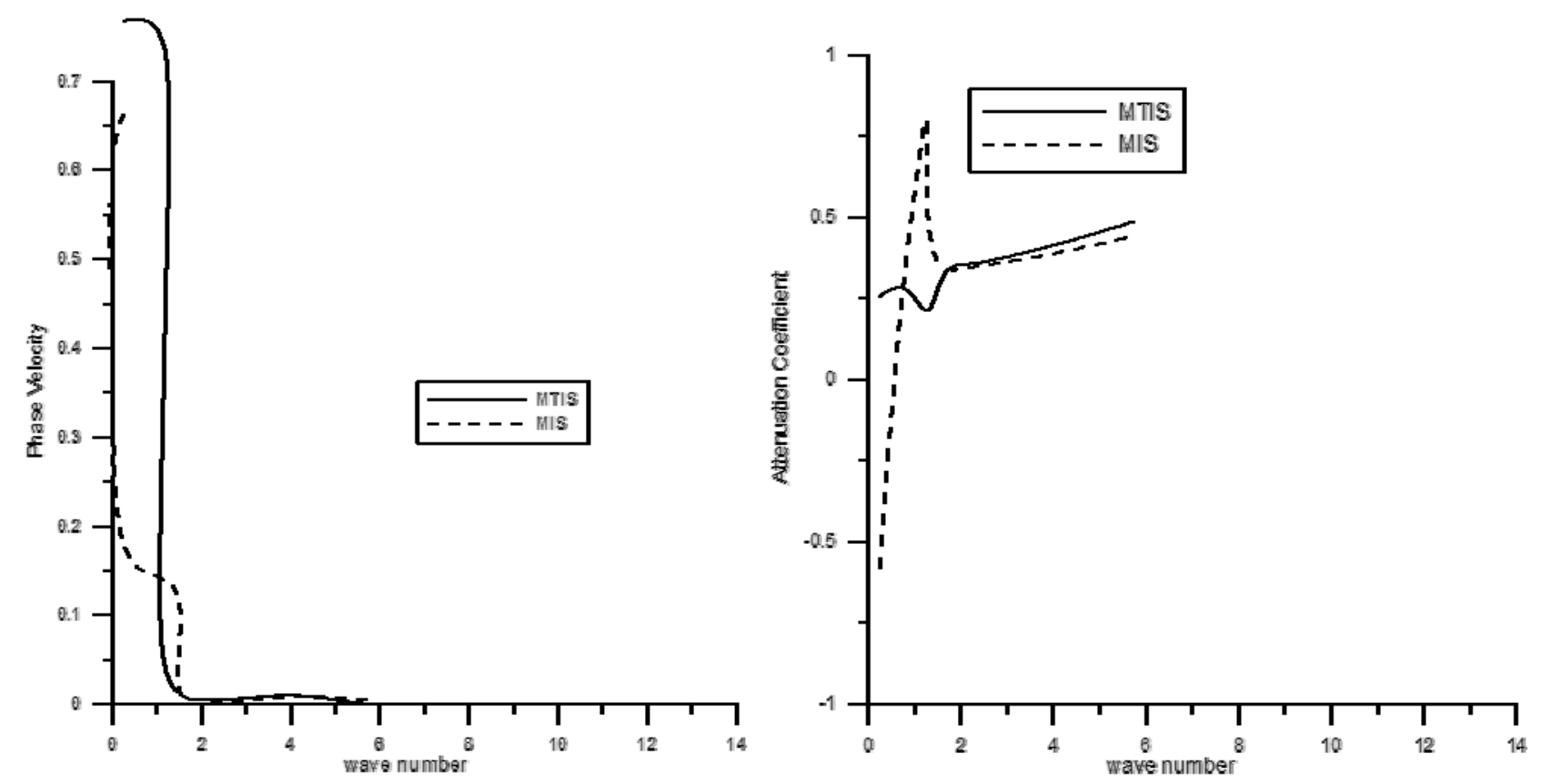

Fig.5. Variation of phase velocity with wave number. Fig.6. Variation of attenuation coefficient with wave number.
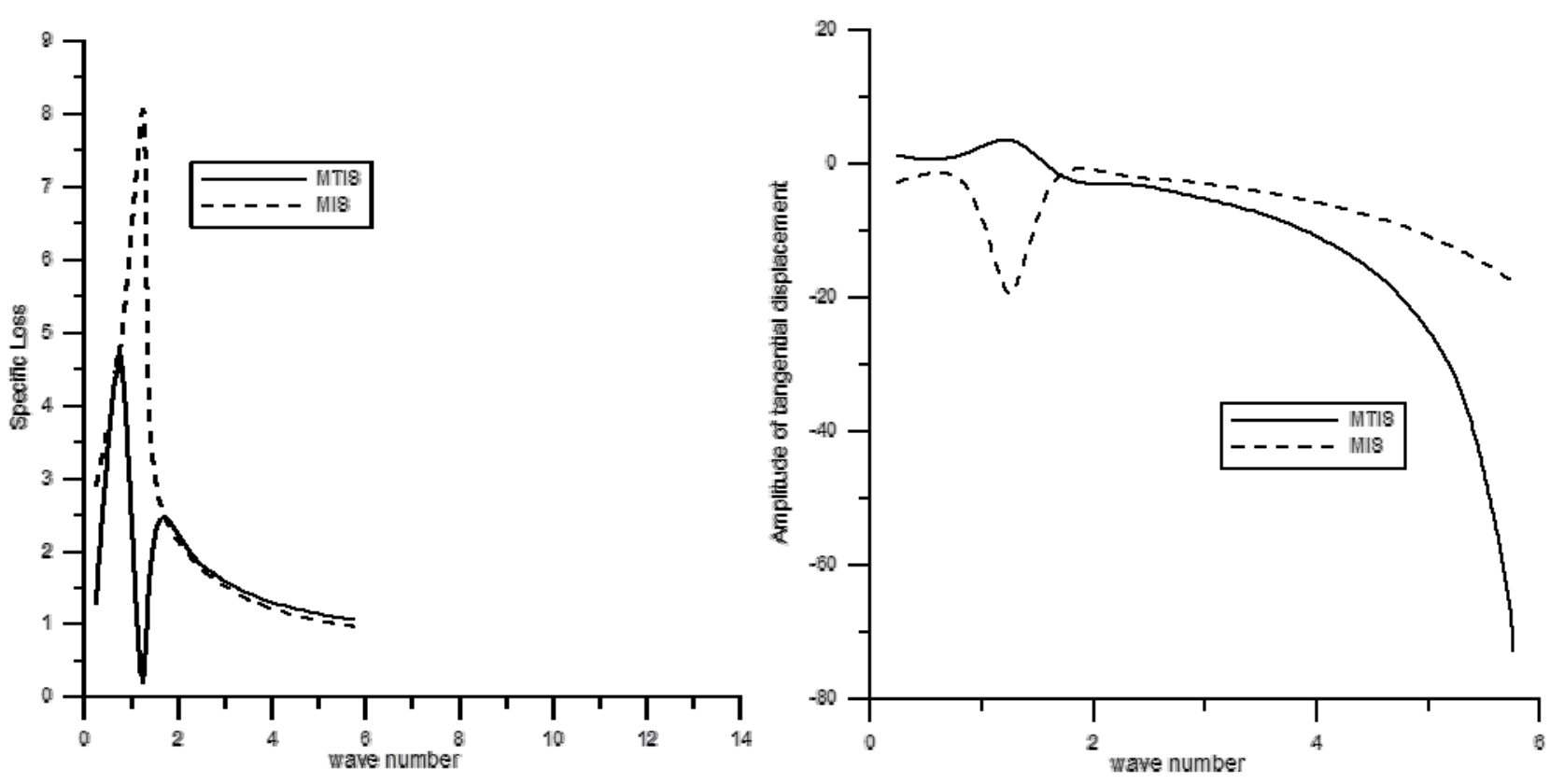

Fig.7. Variation of specific loss with wave number. Fig.8. Variation of amplitude of tangential displacement with wave number. 
It can be deduced from Fig. 5 that the value of phase velocity for MTIS shows a large hump within the interval $(0,2)$, and then becomes constant, while for MIS, its value starts with a sharp decrease and tends to attain a constant value afterwards. The value of the attenuation coefficient for the case of MTIS oscillates initially and then decreases in the end, while the reverse behavior is observed for MIS, as depicted in Fig.6. Figure 7 shows the behavior of the specific loss with the wave number. It is shown that the value of the specific loss initially oscillates and then decreases to attain a constant value for both the cases of MTIS and MIS.

Figures 8-10 indicate the trend of variations of amplitudes of normal displacement, tangential displacement and microrotation with respect to the wave number.

It is noticed from Figs 8 that the amplitude of normal displacement initially decreases and then shows a peak value within the interval $(1,3)$ and then tends to attain a constant value, as far as the case of MTIS is concerned. However, for MIS, its value initially increases, and then sharply decreases to attain a constant value. The amplitude of oscillations for MTIS is higher as compared to this of MIS. From Fig.9, it can be seen that the value of amplitude of tangential displacement starts with a decrease up to the value 5 of the wave number and then sharply increases to become constant. A similar behavior is observed for the case of MIS. Also, it follows from Fig.10 that the value of amplitude of microrotation for both the cases of MTIS and MIS shows a sharp increase within the interval $(.25,1.5)$ and then attains a constant value.

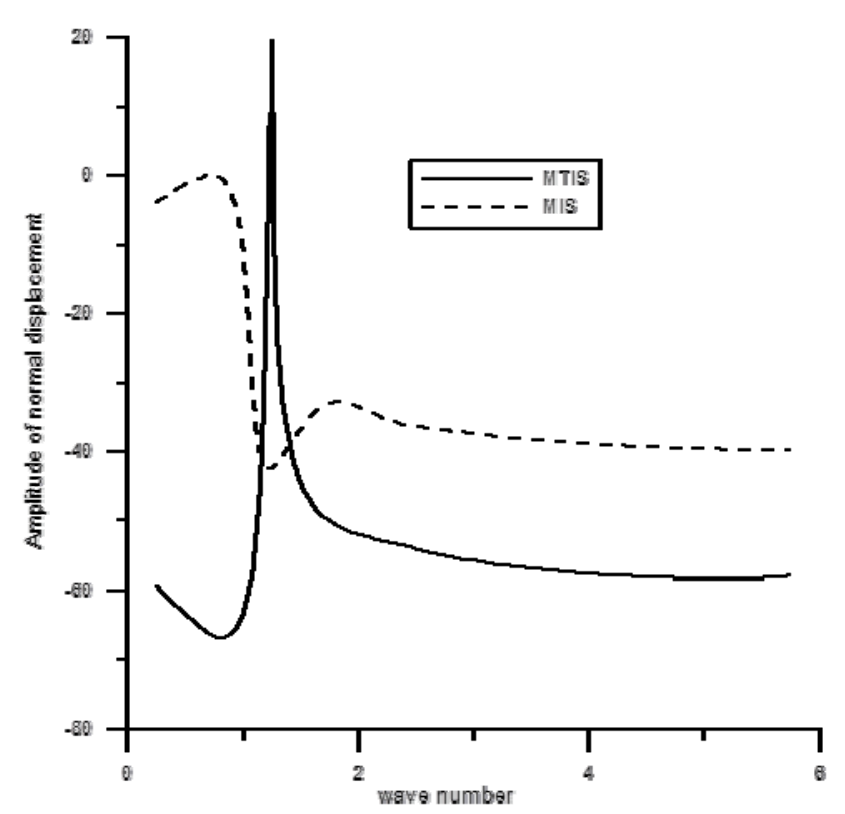

Fig.9. Variation of amplitude of normal displacement with wave number.

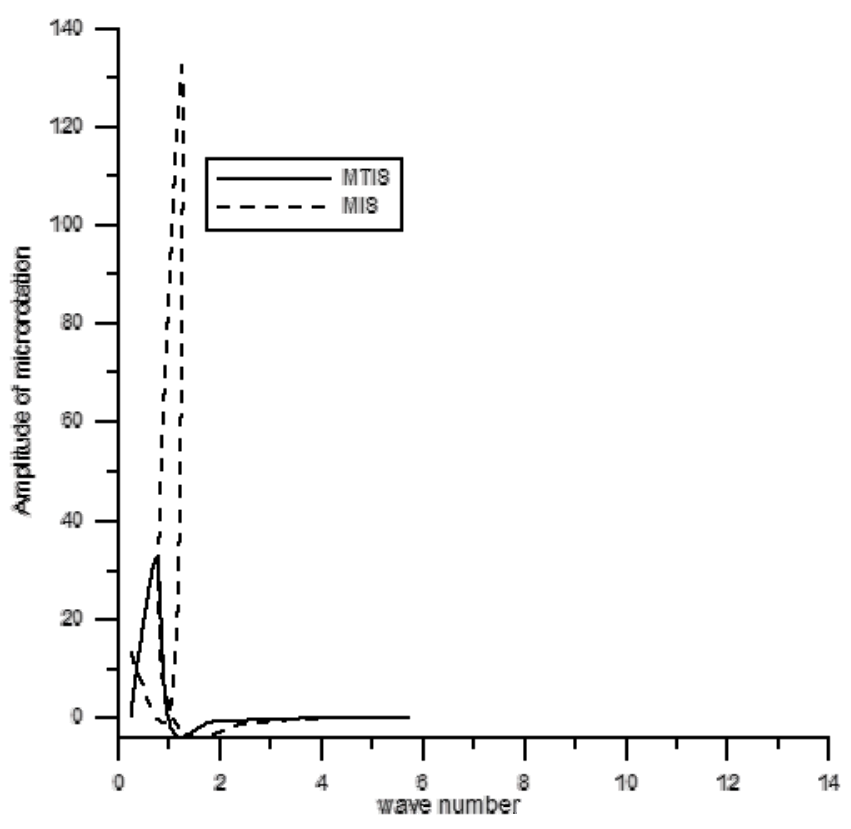

Fig.10. Variation of amplitude of microrotation with wave number.

\section{Conclusions}

The propagation of waves for a micropolar transversely isotropic solid half space underlying the layer of an inviscid liquid is investigated. A significant effect of fluid loading of varying thickness is observed on the phase velocity, attenuation coefficient and specific loss. It is concluded that their values, initially oscillate and then attain a constant value for thickness $H=0.01$, while for $H=1$, the values oscillate with large amplitudes. In the case of the solid half-space, phase velocity and specific loss attain quite large values at vanishing wave number, which sharply flatten out to become steady with increasing wave number. However, the value of attenuation coefficient varies arbitrarily for both the cases of MTIS and MIS. It is also shown in the figures that the values of amplitudes of displacements and microrotation get increased with an increase in anisotropy. 


\section{Nomenclature}

$c^{*} \quad$ - specific heat at constant strain

$j-$ microinertia

$m_{i j} \quad$ - components of couple stress

$t_{i j}$ - components of stress

$u_{i}$ - components of displacement

$\delta_{i j}-$ Kronecker delta

$\lambda_{0} \quad$ - Lame's constant

$\varphi_{i}$ - components of microrotation

$\rho$ - density

$\rho_{0}-$ density of the fluid

$\varphi-$ microrotation vector

$A_{i j k l}, G_{i j k l}, B_{i j k l}$ are characteristic constants of the material following the symmetry properties given by Eringen (1999).

\section{References}

Abubakar I. (1962): Free vibrations of a transversely isotropic plate. - Quart. Journ. Mech. and Applied Math., XV, Pt.I, pp.129-136.

Brulin O. and Hsieh R.K.T. (1981): Mechanics of Micropolar Media. - World Scientific Publishing Corp. Pvt. Ltd..

Eringen A.C. (1966): Linear theory of micropolar elasticity. - J. Math. Mech., vol.16, pp.909-923.

Eringen A.C. (1999): Microcontinuum Field Theories I: Foundations and Solids. - New York: Springer-Verlag.

Gauthier R.D. (1982): In experimental investigations on micropolar media. - Mechanics of Micropolar Media (eds) O. Brulin, RKT Hsieh. (Singapore: World Scientific).

Keck E. and Armenkas A.E. (1971): Wave propagation in transversely isotropic layered cylinders. - J. of the Engg. Mech. Division Procc. of the Amer. Soc. of Civil Engg., EM 2, pp.541-555.

Kolsky H. (1963): Stress Waves in Solids. - Oxford: Clarendon Press; New York: Dover Press.

Mindlin R.D. (1964): Microstructure in linear elasticity. - Arch. Rational Mech. Anal., vol.16, pp.51-78.

Parfitt V.R. and Eringen A.C. (1969): Reflection of plane waves from the flat boundary of a micropolar elastic halfspace. - The Journal of Acoustical Society of America, vol.45, No.5, pp.1258-1272.

Payton R.G. (1992): Wave propagation in a restricted transversely isotropic elastic solid whose slowness surface contains conical points. - [Q. J.] Mech. Appl. Math., vol.45, Pt.2, pp.183-197.

Slaughter W.S. (2002): The Linearized Theory of Elasticity. - Birkhauser.

Suhubi E.S. and Eringen A.C. (1964): Non-linear theory of simple microelastic solids II. - Int. J. Engng. Sci., vol.2, pp.389-404.

Suvalov A.L., Poncelet O., Deschamps M. and Baron C. (2005): Long-wavelength dispersion of acoustic waves in transversely inhomogeneous anisotropic plates. - Wave Motion, vol.42, pp.367-382.

Received: January 30, 2010

Revised: October 27, 2013 